\title{
DYNAMIC ABSORBER FOR SELF-EXCITED OSCILLATION OF THE RECTANGULAR THIN PLATE WITH CREEP
}

\author{
HOANG VAN DA \\ Technical University of Mining and Geology
}

\section{Introduction}

The effect of dynamic absorber for the forced oscillation and parametric one has been investigated $[5,6]$.

In this paper the effect of dynamic absorber for the self-excited oscillation of the rectangular thin plate with creep is studied by means of the asymptotic method for high-order system [1] and boundary value problem [3].

1. Formulation of problem and the equation of motion

Let's consider the self-excited oscillation of a rectangular thin plate with creep, having thickness $h$, Young's modulus $E$, specific mass $\rho$ and length of edges $b, c$, which is supported on four edges.

The plate is loaded by the aerodynamic force in the vertical direction [2]

$$
f(\dot{W})=\varepsilon\left(h_{1} \dot{W}^{1}-h_{2} \dot{W}^{3}\right), \quad h_{1}>0, h_{2}>0 .
$$

To decrease or to damp the oscillation of the plate, we use a weak dynamic absorber as shown in Fig. 1

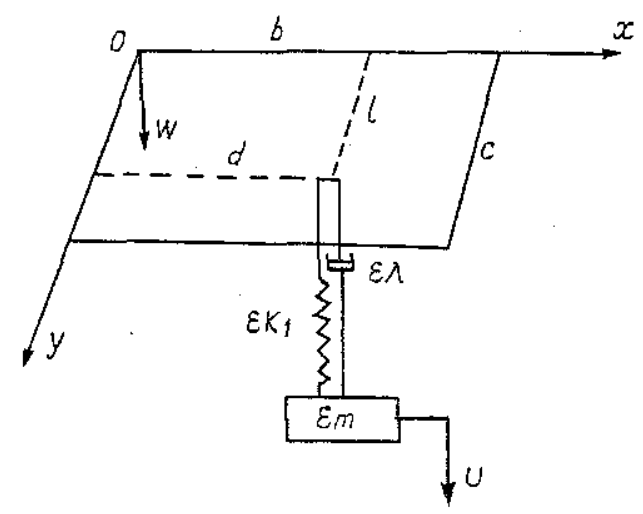

Fig. 1 [5]

The motion of the system under consideration is described by the following equational system

$$
\begin{aligned}
& \frac{\partial^{3} W}{\partial t^{3}}+\xi \frac{\partial^{2} W}{\partial t^{2}}+\frac{D_{1}}{M} \frac{\partial}{\partial t}\left(\nabla^{4} W\right)+\xi D_{1} \nabla^{4} W \\
& =\varepsilon \frac{\xi}{M}\left\{K_{1}[U-W(d, \ell, t)] \delta(x-d) \delta(y-\ell)+\lambda\left[\frac{d U}{d t}-\frac{\partial}{\partial t} W(d, \ell, t)\right] \delta(x-d) \delta(y-\ell)+f(\dot{W})\right\}
\end{aligned}
$$




$$
\begin{aligned}
& +\frac{\varepsilon}{M} \frac{\partial}{\partial t}+\left\{K_{1}[U-W(d, \ell, t)] \delta(x-d) \delta(y-\ell)\right. \\
& \left.+\lambda\left[\frac{d U}{d t}-\frac{\partial}{\partial t} W(d, \ell, t)\right] \delta(x-d) \delta(y-\ell)+f(W)-D_{1} \frac{E_{2}}{E_{1}} \frac{\partial \nabla^{4} W}{\partial t}\right\} \\
& \quad \quad \frac{m^{2} U}{d t^{2}}+K_{1}[U-W(d, \ell, t)]=-\lambda\left[\frac{d U}{d t}-\frac{\partial}{\partial t} W(d, \ell, t)\right]
\end{aligned}
$$

where $W=W(x, y, t)$ is the deflection of the plate, $\gamma$-Poisons's ratio.

$$
\begin{aligned}
& \nabla^{4}=\left(\frac{\partial^{4}}{\partial x^{4}}+2 \frac{\partial^{4}}{\partial x^{2} \partial y^{2}}+\frac{\partial^{4}}{\partial y^{4}}\right)-\text { Laplace's operator, } \\
& D_{1}=\frac{E_{1} h^{3}}{12\left(1-\gamma^{2}\right)}, \quad \xi=\frac{E_{2}}{K_{2}}
\end{aligned}
$$

$E_{1}, E_{2}, K_{2}$ are the constants characteristing the properties of material $[4], M=h \rho, \rho$ is specific mass, $\varepsilon$ is a small parameter.

The relevant homogeneous boundary conditions are

$$
\begin{aligned}
& \left.W\right|_{x=0, b}=0, \quad \frac{\partial^{2} W}{\partial x^{2}}+\left.\gamma \frac{\partial^{2} W}{\partial y^{2}}\right|_{x=0, b}=0, \\
& \left.W\right|_{y=0, c}=0, \quad \frac{\partial^{2} W}{\partial y^{2}}+\left.\gamma \frac{\partial^{2} W}{\partial x^{2}}\right|_{y=0, c}=0 .
\end{aligned}
$$

For simplicity, it is supposed that $M=1$. By setting $\Omega^{2}=\frac{D_{1}}{M}, \omega^{2}=\frac{K_{1}}{m}$, the equational system (1.2), (1.3) is written in the form

$$
\begin{gathered}
\frac{\partial^{3} W}{\partial t^{3}}+\xi \frac{\partial^{2} W}{\partial t^{2}}+\frac{\partial}{\partial t}\left(\Omega^{2} \nabla^{4} W\right)+\xi \Omega^{2} \nabla^{4} W=\varepsilon F\left(U, \frac{d U}{d t}, W, \frac{\partial W}{\partial t}, \ldots\right), \\
\frac{d^{2} U}{d t^{2}}+\omega^{2}[U-W(d, \ell, t)]=-\frac{\lambda}{m}\left[\frac{d U}{d t}-\frac{\partial}{\partial t} W(d, \ell, t)\right],
\end{gathered}
$$

\section{Construction of the asymptotic solution}

The particular solution of the equation (1.2) with the boundary condition (1.5) can be found in the following form

$$
\begin{aligned}
& W(x, y, t)=S_{n m}(t) Z_{n m}(x, y), \\
& Z_{n m}(x, y)=\sin \frac{n \pi x}{b} \sin \frac{m \pi y}{c} .
\end{aligned}
$$

Substituting (2.1) in the equation (1.5) and applying Galerkin - Bubnov's method, we obtain the equations for unknown functions $S(t), U(t)$

$$
\begin{gathered}
\dddot{S}_{n m}(t)+\xi \ddot{S}_{n m}(t)+\Omega_{n m}^{2} \dot{S}_{n m}(t)+\xi \Omega_{n m}^{2} S_{n m}(t)=\varepsilon F(t), \\
\frac{d^{2} U}{d t^{2}}+\omega^{2}\left[U-S_{n m}(t) Z_{n m}(d, \ell)\right]=-\frac{\lambda}{m}\left[\frac{d U}{d t}-\dot{S}_{n m} Z_{n m}(d, \ell)\right],
\end{gathered}
$$

where

$$
\Omega_{n m}^{2}=\Omega^{2}\left[\left(\frac{n \pi}{b}\right)^{2}+\left(\frac{m \pi}{c}\right)^{2}\right]^{2}
$$




$$
\begin{aligned}
F(t)= & \frac{4 \xi}{b c}\left\{K_{1}\left[U-S_{n m}(t) Z_{n m}(d, \ell)\right] Z_{n m}(d, \ell)+\lambda\left[\frac{d U}{d t}-\dot{S}_{n m}(t) Z_{n m}(d, \ell)\right] Z_{n m}(d, \ell)\right. \\
& \left.+\frac{b c}{4}\left[h_{1} \dot{S}_{n}(t)-h_{3} \dot{S}_{n}^{3}(t)\right]\right\}+\frac{4}{b c} \frac{\partial}{\partial t}\left\{K_{1}\left[U-S_{n m}(t) Z_{n m}(d, \ell)\right] Z_{n m}(d, \ell)\right. \\
& \left.+\lambda\left[\frac{d U}{d t}-\dot{S}_{n m}(t) Z_{n m}(d, \ell)\right] Z_{n m}(d, \ell)+\frac{b c}{4}\left[h_{1} \dot{S}_{n m}(t)-\frac{9 h_{3}}{16} \dot{S}_{n m}^{3}(t)\right]\right\} \\
& -\frac{E_{2}}{E_{1}} \Omega_{n m}^{2} \dot{S}_{n m}(t)
\end{aligned}
$$

The partial solution of the equational system (2.2), (2.3) is found in the following form [2]

$$
\begin{aligned}
S_{n m}(t) & =a \cos \varphi, \quad \varphi=\left(\Omega_{n m} t+\Psi\right), \\
U & =a(L \cos \varphi+N \sin \varphi),
\end{aligned}
$$

where $L, N$ are constants and the variables $a, \Psi$ are determined from the differential equational system

$$
\begin{aligned}
& \frac{d a}{d t}=\varepsilon A_{1}(a)+\varepsilon^{2} A_{2}(a)+\ldots \\
& \frac{d \Psi}{d t}=\varepsilon B_{1}(a)+\varepsilon^{2} B_{2}(a)+\ldots
\end{aligned}
$$

By substituting (2.6), (2.7) into the equation (2.3) we get after simple calculations

$$
\begin{aligned}
& L=\frac{\left[\Omega^{2}\left(\omega^{2}-\Omega_{n m}^{2}\right)+\frac{\lambda^{2}}{m^{2}} \Omega_{n m}^{2}\right] Z_{n m}(d, \ell)}{\left[\left(\omega^{2}-\Omega_{n m}^{2}\right)^{2}+\frac{\lambda^{2}}{m^{2}} \Omega_{n m}^{2}\right]}, \\
& N=\frac{\frac{\lambda}{m} \Omega_{n m}^{2} Z_{n m}(d, \ell)}{\left[\left(m^{2}-\Omega_{n m}^{2}\right)^{2}+\frac{\lambda^{2}}{m^{2}} \Omega_{n m}^{2}\right]} .
\end{aligned}
$$

The quantities $A_{i}, B_{i}$ are determined from the following expressions [5]

$$
A_{i}=-\frac{\left(\Omega_{n m} G+\xi H\right)}{2 \Omega_{n m}\left(\Omega_{n m}^{2}+\xi^{2}\right)}, \quad B_{i}=-\frac{\left(\xi G-\omega_{n m} H\right)}{2 a \Omega_{n m}\left(\omega_{n m}^{2}+\xi^{2}\right)},
$$

where

$$
G=\frac{1}{\pi} \int_{0}^{2 \pi} F \cos \varphi d \varphi, \quad H=\frac{1}{\pi} \int_{0}^{2 \pi} F \sin \varphi d \varphi .
$$

After simple calculations we obtain

$$
\begin{aligned}
2 \Omega_{n m} \frac{d a}{d t} & =\varepsilon\left[-k_{1} R-\lambda \Omega_{n m} S-\xi \Omega_{n m} P+h_{1} \Omega_{n m}-\frac{9}{16} h_{3} a^{2} \Omega_{n m}^{3}\right] a \\
2 a \Omega_{n m} \frac{d \Psi}{d t} & =\varepsilon\left[K_{1} S-\lambda \Omega_{n m} R+\Omega_{n m}^{2} P\right]
\end{aligned}
$$

where

$$
R=\frac{2 N}{b c} Z_{n m}(d, \ell), \quad S=\frac{2}{b c}\left[Z_{n m}(d, \ell)-L\right] Z_{n m}(d, \ell), \quad P=\frac{E_{2} \Omega_{n m}^{2}}{2 E_{1}\left(\Omega_{n m}^{2}+\xi^{2}\right)} .
$$


Substituting the quantities (2.12) into the equational system (2.11), we get

$$
\begin{aligned}
& 2 \frac{d a}{d t}=\varepsilon\left[-\lambda \frac{2 \Omega_{n m}^{4} Z_{n m}^{2}(d, \ell)}{b c\left[\left(\omega^{2}-\Omega_{n m}^{2}\right)^{2} \frac{\lambda^{2}}{m^{2}} \Omega_{n m}^{2}\right]}-\xi P+h_{1}-\frac{9}{16} h_{3} a^{2} \Omega_{n m}^{2}\right] a \\
& 2 a \frac{d \psi}{d t}=\varepsilon\left[\frac{2}{b c} \frac{\Omega_{n m} Z_{n m}^{2}(d, \ell)}{\left[\left(\omega^{2}-\Omega_{n m}^{2}\right)^{2}+\frac{\lambda^{2}}{m^{2}} \Omega_{n m}^{2}\right]}+\Omega_{n m} P\right]
\end{aligned}
$$

From (2.13) it is easy to see that if

$$
h_{1}<\lambda \frac{2 \Omega_{n m}^{4} Z_{n m}^{2}\left(d_{s} \ell\right)}{b c\left[\left(\omega^{2}-\Omega_{n m}^{2}\right)^{2}+\frac{\lambda^{2}}{m^{2}} Q_{n m}^{2}\right]}+\xi P
$$

the equilibrium $a=\dot{a}=0$ is stable and the mechanical system under consideration is at rest. If

$$
h_{1}>\lambda \frac{2 \Omega_{n m}^{4} Z_{n m}^{2}(d, \ell)}{b c\left[\left(\omega^{2}-\Omega_{n m}^{2}\right)^{2}+\frac{\lambda^{2}}{m^{2}} \Omega_{n m}^{2}\right]}+\xi P
$$

the equilibrium $a=\dot{a}=0$ is unstable and there exists a stationary self-excited oscillation with the amplitude $a_{0}$ determined by

$$
A_{0}^{2}=\frac{9}{16} h_{3} a_{0}^{2}=h_{1}-\left[\lambda \frac{2 \Omega_{n m}^{2} Z_{n m}^{2}(d, \ell)}{b c\left[\left(\omega^{2}-\Omega_{n m}^{2}\right)^{2}+\frac{\lambda^{2}}{m^{2}} \Omega_{n m}^{2}\right]}+\xi \frac{P}{\Omega_{n m}^{2}}\right],
$$

if the right-hand side of (2.16) is positive. In Fig. 2 the dependence of amplitude of oscillation on the damping parameter $\lambda$ is presented for the case $h_{1}=0.05 ; \Omega_{n m}^{2}=1 ; \omega^{2}=0.8 ; P=0.1$; $\xi=0.1 ; b=1.5 ; c=1.2$.

The curve 1 corresponds to $Z_{n m}=0.2$ and the curve $2-$ to $Z_{n m}=0.15$. When $\omega, \Omega_{n m}$ are in equality, the expression (2.16) takes the form:

$$
A_{0}^{2}=\frac{9}{16} h_{3} a_{0}^{2}=h_{1}-\left[\frac{2 \Omega_{n m}^{2} Z_{n m}^{2}(d, \ell)}{\left[b c \frac{\lambda}{m^{2}} \Omega_{n m}^{2}\right]}+\frac{\xi P}{\Omega_{n m}^{2}}\right],
$$

and the resonance curve is shown in Fig. 2 by " 0 ". In this case the effect of quenching of the absorber is highest.

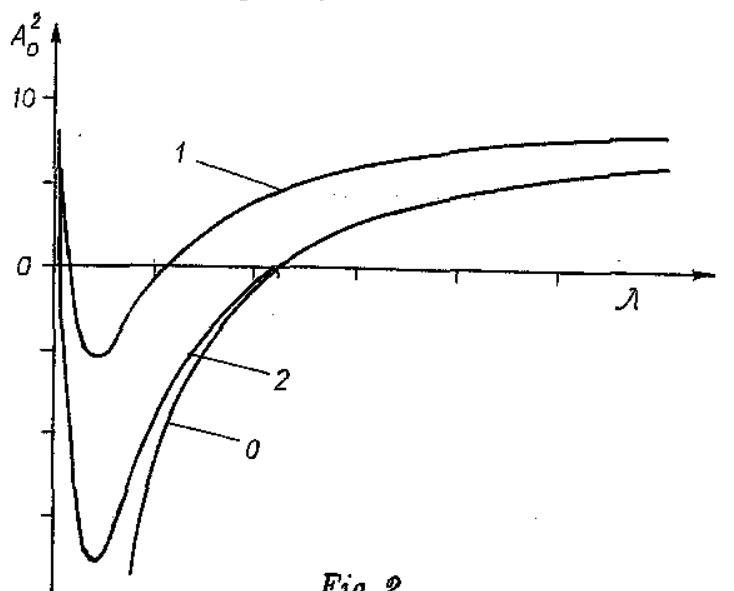

Fig. 2 


\section{Conclusion}

1. The equation of motion for a rectangular thin plate with creep, combined a weak dynamic absorber was set up. Its solution has been found by means of the asymptotic method.

2. The effect of the weak dynamic absorber has been investigated. It was shown that the damping force $(\lambda)$ plays an important role in quenching the self-excited oscillation by means of dynamic absorber.

3. From (2.16), it is easy to see that the effect of the absorber will be high, if

$$
Z_{n m}(d, \ell)=\sin \frac{n \pi d}{b} \sin \frac{m \pi \ell}{c}=1 \quad \text { or if } \quad d=\frac{b}{2 n}, \quad \ell=\frac{c}{2 m} .
$$

In particular case $n=1, m=1$, we have

$$
d=\frac{b}{2} ; \quad \ell=\frac{c}{2}
$$

This publication is completed with the financial support from the National Basic Research Program in Natural Sciences.

\section{References}

1. Nguyen Van Dao. Non-linear oscillation of high order systems. NCSR of Vietnam, Hanoi 1979.

2. Mitropolski Yu. A., Nguyen Van Dao. Applied asymptotic methods in nonlinear oscillations, Hanoi 1994.

3. Mitropolski Yu. A., Masinkov. asymptotic solution in partial derivative equation, Kiev 1976.

4. Rgianhisun A. R. Theory of creep, Moscow 1966.

5. Le Van Co, Hoang Van Da, Tran Dinh Son. Dynamic obsorber for linear forced oscillations of the rectangular thin plate with creep. Proceedings of the fourth national conference on solid mechanics, Hanoi 1994.

6. Hoang Van Da. Dynamic absorber for parametric oscillations of the rectangular thin plate with creep on elastic foundation. Journal of Mechanics, NCNST of Vietnam, T.XVIII, No2, 1996.

Received June 3, 1996

\section{Bộ TA̛T CHẤN ĐộNG LỰC ĐỐI VớI DAO ĐộNG TỤ CHẤN CƯA BÁ̀N MỎNG CHŨ NHÂT TƯ BIẾN}

Trong bài báo này, tác giả khảo sát hiệu lực của bộ tắt chấn động lực đối vói dao động tự chấn cưa bản mơng chữ nhật từ biến. Kết quả cho thấy răng nếu $h_{1}$ thớa mãn (2.15) thì tồn tại dao động tự chẩn quanh vị trí cân băng không ổn định. Hệ số cân $\lambda$ của bộ tăt chấn có vai trò quan trọng trong việc giăm biên độ dao động. Tuy nhiên dao động tự chấn vẫn không bị dập tắt hoàn toàn. 